\title{
SAÚDE MENTAL NA ATENÇÃO PRIMÁRIA À SAÚDE: PERCEPÇÕES DA EQUIPE DE SAÚDE DA FAMÍLIA
}

\author{
Geslaney Reis da Silva ${ }^{1}$, Helca Franciolli Teixeira Reis ${ }^{2}$, Edirlei Machado Dos-Santos ${ }^{3}$, Marcos Paulo Almeida \\ Souza ${ }^{4}$, Renata Lessa Azevedo ${ }^{5}$
}

\begin{abstract}
RESUMO: Objetivou-se conhecer a percepção dos profissionais de saúde da família acerca da implementação de ações de saúde mental na atenção primária à saúde. Pesquisa qualitativa, com dados coletados em entrevistas semiestruturadas e analisadas pela técnica análise de conteúdo temática, no período de abril a junho de 2013. Participaram 17 trabalhadores da Estratégia Saúde da Família do município de Vitória da Conquista, Bahia. Depreenderam-se três categorias: Percepção acerca da promoção da saúde mental na atenção primária à saúde; Dificuldades/Limitações para implementação de ações de saúde mental na Estratégia Saúde da Família; Estratégias/ Intervenções para implementação de saúde mental na atenção primária à saúde. Os participantes apresentaram dificuldades em relatar ações de promoção à saúde mental e demonstraram fragilidades na produção do cuidado. Observou-se a percepção deles em relação à necessidade de ampliação dos processos formativos em saúde mental, com vistas ao aprimoramento das ações em saúde mental na atenção primária à saúde.
\end{abstract}

DESCRITORES: Saúde mental; Saúde da família; Atenção primária à saúde; Enfermagem.

\section{MENTAL HEALTH IN PRIMARY CARE: PERCEPTIONS OF THE FAMILY HEALTH CARE TEAM}

ABSTRACT: The present study aimed to obtain knowledge on the perception of family health care teams regarding the implementation of mental health actions in primary care. Qualitative research, with data collected in semi-structured interviews and analyzed by thematic content analysis from April to June 2013. The study included the participation of 17 workers of the Family Health Strategy of the city of Vitória da Conquista, Bahia.Three categories emerged: Perception of mental health promotion in primary health care; Difficulties /Limitations to implement mental health care actions under Brazil's Family Health Strategy; Strategies/Interventions regarding the implementation of mental health in primary health care.The participants found it difficult to report actions targeted to the promotion of mental health and exposed the weaknesses in health production. They perceived the need to expand mental health trainingprocesses, in order to improve mental health actions developed in primary health care.

DESCRIPTORS: Mental health; Family health; Primary health care; Nursing.

\section{SALUD MENTAL EN LA ATENCIÓN PRIMARIA A LA SALUD: PERCEPCIONES DEL EQUIPO DE SALUD DE LA FAMILIA}

RESUMEN: Fue objetivo del estudio conocer la percepción de los profesionales de salud de la familia acerca de la implementación de acciones de salud mental en la atención primaria a la salud. Investigación cualitativa, cuyos datos fueron obtenidos en entrevistas semiestructuradas y analizados por la técnica de análisis de contenido temático, en el periodo de abril a junio de 2013. Participaron 17 trabajadores de la Estrategia Salud de la Familia del municipio de Vitória da Conquista, Bahia. Resultaron tres categorías: Percepción acerca de la promoción de la salud mental en la atención primaria a la salud; Dificultades/limitaciones para implementación de acciones de salud mental en la Estrategia Salud de la Familia; Estrategias/intervenciones para implementación de salud mental en la atención primaria a la salud. Los participantes presentaron dificultades para relatar acciones de promoción a la salud mental y demostraron fragilidades en la producción del cuidado. Se observó la percepción de ellos acerca de la necesidad de ampliación de los procesos de formación en salud mental, confines de perfeccionar las acciones en salud mental en la atención primaria a la salud.. DESCRIPTORES: Salud mental; Salud de lafamilia; Atención primaria a lasalud; Enfermería.

1Enfermeiro. Mestrando em Enfermagem e Saúde. Enfermeiro da Unimed do Sudoeste. Vitória da Conquista, BA, Brasil. Enfermeira. Mestre em Enfermagem e Saúde. Docente da Universidade Federal da Bahia. Vitória da Conquista, BA, Brasil. ${ }^{3}$ Enfermeiro. Doutor em Enfermagem. Docente da Universidade Federal da Bahia. Vitória da Conquista, BA, Brasil. ${ }^{4}$ Enfermeiro. Mestre em Ciências Fisiológicas. Universidade Federal da Bahia. Vitória da Conquista, BA, Brasil. ${ }^{5}$ Enfermeira. Residente em Saúde Mental. Universidade Federal de Sergipe. Vitória da Conquista, BA, Brasil. 


\section{INTRODUÇÃO}

No final da década de 1970 e início dos anos de 1980, concomitantemente à Reforma Sanitária Brasileira (RSB), ocorreu o Movimento da Reforma Psiquiátrica Brasileira (RPB), o qual se inspirou nos ideais pioneiros da Psiquiatria Democrática Italiana, cujo alicerce fundamentou-se em um novo saber psiquiátrico, contrário às práticas manicomiais e à política do abandono, rumo à desinstitucionalização da Pessoa com Sofrimento Mental (PSM) ${ }^{(1)}$.

Destarte, a RSB corroborou para a efetivação dos fundamentos preconizados pela RPB, por possuir a integralidade e a universalidade como principais pontos teóricos para o acolhimento das PSM, não apenas no nível secundário e terciário, mas também na Atenção Primária à Saúde (APS).

De maneira semelhante, a RPB tem buscado responsabilização política, bem como a (re)incorporação das práticas de acolhimento aos usuários com demanda de saúde mental nos diversos níveis da atenção à saúde, em especial na APS ${ }^{(1)}$. Tais mudanças no modelo assistencial à saúde mental, antes com foco na doença e no hospital psiquiátrico e agora com ênfase na pessoa que sofre, incorpora a família como protagonista na produção do cuidado a PSM, sendo a comunidade compreendida como cenário para reinserção social ${ }^{(2)}$.

Nesta perspectiva, entende-se que a família, sobretudo na fase inicial do sofrimento mental, pode atuar de forma incisiva na formulação de uma nova trajetória de vida para todos envolvidos nesse processo, porém seus recursos emocionais, temporais, econômicos e seus saberes carecem de uma melhor orientação, a partir da contribuição dos trabalhadores de saúde ${ }^{(3-4)}$.

Ademais, os trabalhadores de saúde que atuam próximos à comunidade, a exemplo da Estratégia de Saúde da Família (ESF), possuem um vínculo construído com as famílias de sua área adstrita, assim a ESF apresenta-se como importante dispositivo para a promoção da saúde mental e enfrentamento de relevantes problemas de saúde pública, como o sofrimento mental e os agravos vinculados ao uso abusivo de álcool e outras drogas ${ }^{(5)}$.

Portanto, a ESF, inserida na própria comunidade, é capaz de desenvolver ações de saúde direcionadas para as necessidades da população, pois conhece e vivencia esta mesma realidade.

Nesse cenário, os trabalhadores que atuam naESF, ao articularem-se no território, possuem condições de estabelecerem parcerias de maneira a utilizar alguns recursos existentes na própria comunidade, tais como: rede de APS, equipamentos sociais, familiares, para em conjunto formarem a rede de apoio social e conseguirem implementar a promoção da saúde mental, com vistas ao alcance do bem estar coletivo, uma vez que a ESF caracteriza-se como dispositivo relevante no campo da atenção à saúde mental.

Diante do exposto, foi elaborado como questão norteadora: Qual a percepção dos profissionais da ESF sobre a implementação de ações da saúde mental na atenção primária? No intuito de responder ao problema de pesquisa apontado, elegeu-se como objetivo do presente estudo, conhecer a percepção dos profissionais de saúde da família acerca da implementação de ações de saúde mental na atenção primária à saúde.

\section{- MÉTODO}

Trata-se de um estudo com abordagem qualitativa, descritiva e exploratória. A pesquisa qualitativa preocupa-se com um nível de realidade que não pode ser quantificado, trabalha com um universo de significados, motivos, aspirações, crenças, valores e atitudes, o que engloba espaço mais profundo de relações, de processos e de fenômenos ${ }^{(6)}$.

O cenário de pesquisa foi composto por uma Estratégia Saúde da Família (ESF) do município de Vitória da Conquista, Bahia. Foram utilizados como critérios de inclusão: ESF vinculada a estágios supervisionados e aulas práticas do Curso de Enfermagem da Universidade Federal da Bahia, por meio do Instituto Multidisciplinar em Saúde; dispor da composição mínima de equipe preconizada pelo Ministério da Saúde e; que todos os trabalhadores da equipe atuassem na mesma equipe por, no 
mínimo, seis meses.

A amostra empregada para o desenvolvimento da pesquisa foi do tipo intencional, pois esta é baseada no conhecimento sobre a população e o propósito do estudo, ou seja, por considerar que os elementos dos indivíduos selecionados possuíam características típicas ou representativas da população(6).

Para a coleta de dados empregou-se a técnica de entrevista semiestruturada. Para isso, foi utilizado um roteiro com a finalidade de subsidiar a pesquisa e estimular os participantes a falarem livremente sobre o objeto de estudo a partir de uma linha de raciocínio e experiências(6). O período de coleta de dados ocorreu entre abril e junho de 2013.

A técnica utilizada para a análise dos dados foi a Análise de Conteúdo na modalidade Temática, que tem como objetivo explicar por meio de inferências qualitativas, as mensagens, os dados coletados, enumerando-os e organizando-os, no intuito de dar sentido às suas características ${ }^{(6)}$.

O projeto de pesquisa foi encaminhado ao Comitê de Ética em Pesquisa do Instituto Multidisciplinar em Saúde da Universidade Federal da Bahia (UFBA), onde foi aprovado mediante parecer de número 301.091.

Com o intuito de garantir a privacidade dos participantes da pesquisa, eles foram identificados por codinomes referentes a nomes de cores: Vermelho, Preto, Verde, Amarelo, Azul, Branco, Cinza, Rosa, Marrom, Bronze, Laranja, Roxo, Bege, Lilás, Dourado, Prata, Castanho.

\section{- RESULTADOS}

A amostra consistiu de 17 trabalhadores que compõe uma equipe de saúde da família (Médico, Enfermeiro, Técnicos de Enfermagem e Agentes Comunitários de Saúde), dos quais quinze eram do sexo feminino e dois do sexo masculino. Referente ao tempo de trabalho, dois participantes possuíam menos de um ano de atuação na ESF, quatro oscilaram entre um e cinco anos, e onze encontravam-se atuando em um tempo superior a cinco anos.

Desse modo, a estratificação da população geral da equipe ESF, a qual concedeu a entrevista, consistiu em uma médica, uma enfermeira, quatro técnicos de enfermagem e onze agentes comunitários de saúde.

A referida ESF possui equipe completa para atuação no seu território de abrangência e está inserida num contexto territorial favorável para sua atuação, pois se encontra em um meio residencial e que também possui instituições sociais para suporte das suas ações na comunidade tais como: igrejas, associação de moradores, escolas públicas e privadas e um Instituto Federal (com ensino do segundo e terceiro grau de escolaridade).

A partir da análise dos dados, foram depreendidas três categorias por meio dos recortes temáticos que se encontram apresentados no Quadro 1.

\section{- DISCUSSÃO}

\section{Categoria Temática 1 - Percepção acerca da promoção da saúde mental na Atenção Primária à Saúde.}

A concepção de promoção à saúde mental por parte da maioria dos trabalhadores entrevistados esteve restrita a determinadas ações acolhedoras e voltadas, unicamente, às pessoas já em sofrimento mental, tais como a escuta da demanda do usuário, orientações generalistas sobre suas necessidades e encaminhamentos para unidades especializadas, como o Centro de Atenção Psicossocial (CAPS).

Além disso, foi mencionado nas falas o receio do atendimento e acolhimento aos usuários em sofrimento mental, por trazer a carga histórica do "louco descontrolado" para as suas atividades laborais. Prejudicando, desta forma, a oportunidade de aproximação e ganho de confiança do usuário para com a equipe. 


\section{Categorias Temáticas}

Percepção acerca da promoção da saúde mental na Atenção Primária à Saúde

\section{Recortes Temáticos}

[...] a gente tenta fazer o acolhimento a eles [pessoas com diagnóstico de transtorno mental], atendendo na condição que temos aqui na Unidade [...]. (VERMELHO)

[...] tem que ter o acolhimento, agora não temos o suporte suficiente né, só a capacitação que nós temos e aí a gente atende [...]. (BRANCO)

[...] é necessário né, porque chegam muitos pacientes pra gente, às vezes quando eles [PSM] chegam a gente tem assim, até certo receio de como trabalhar, o que pode fazer, o que não pode fazer. (PRETO)

Eu vejo assim, talvez uma deficiência em acolher esse paciente, talvez a gente acabe não sabendo lidar, mas não porque nós não estamos preparados, mas a gente não saber como esse paciente vai agir [...]. (AMARELO)

[...] Quando fala em promover a saúde mental, promover um bem estar mental mesmo, não só aquela pessoa que está doente, mas pro seu familiar e para as pessoas que poderiam vir a adoecer. Então é promover a saúde mental como a própria palavra diz não a doença, né? (DOURADO)

Dificuldades e limitações para a implementação de ações de saúde mental nesta Estratégia Saúde da Família

Estratégias/ intervenções para implementação de saúde mental na Atenção Primária à Saúde
Existem porque, nem todos os profissionais da saúde, principalmente do caso da atenção primária, tem conhecimento ou habilidade pra tratar desse assunto [...] (LARANJA).

[...] é preciso ter uma capacitação para todos os profissionais, não só enfermeiro e médico, como dos técnicos, agente comunitários de saúde, SAME [Serviço de Arquivo Médico e Estatística], vigilantes, em geral, pra até identificar e saber lidar com esses pacientes. (LILÁS)

Sim, a primeira dificuldade da gente, é em relação a nossa formação. Saúde mental é muito ampla, é muito complexa. A gente não tem a formação voltada como deveria, e uma capacitação que a gente recebeu foi muito pouca, porém de uma semana, pra trabalhar com a saúde mental [...]. (DOURADO)

[...] falta mesmo é profissional nesse sentido mesmo, profissional que entenda mais e que participe mais com a gente, porque às vezes a gente detecta na área, mas não tem como trazer esse paciente pra unidade [...]. (ROXO)

Eu acho que a limitação é somente a capacitação. A gente já teve um período de sensibilização aqui, mas se você capacita, porque todo mundo diz assim: Eu não faço porque eu não conheço. Então se você capacita, eu acho que o problema desaparece, porque as pessoas têm medo dos pacientes com doença mental, né? As pessoas têm medo, então esse medo é preconceito, e a gente precisa acabar com isso. E como é que acaba com isso? Com a educação, educação continuada. (PRATA)

A estratégia que a gente utiliza hoje é fazendo os grupos [...]. (CINZA)

[...] fazemos também um trabalho de terapia comunitária no bairro, onde damos um apoio às famílias e as pessoas que sentem algum sofrimento mental. (ROSA)

Aqui nessa Unidade, em especial, a gente tem um trabalho que se chama Terapia Comunitária, Roda de Terapia. Então a Roda de Terapia é um espaço de escuta, onde as pessoas partilham sofrimentos [...]. (PRATA)

Nós fazemos grupos de terapias, temos o grupo terapia comunitária. Também fazemos as visitas domiciliares onde identificamos aqueles pacientes com problemas de saúde mental [...]. (BRONZE)

Nós formamos grupos pra essas pessoas, fazendo com que elas venham até esse grupo, e nesse grupo elas têm um espaço pra falar e pra discutir o seu problema, além da gente tentar trazer também a família desses pacientes mostrando pra eles essa realidade do paciente com o problema mental. (LARANJA)

A maioria dos participantes da pesquisa ancoraram seus relatos na promoção da saúde mental com ênfase na abordagem às pessoas com diagnósticos de transtorno mental, pautando-se no modelo biomédico, e não associaram a importância de promover saúde mental aos usuários da ESF de uma forma geral, independente de um quadro de sofrimento mental instalado( ${ }^{(7)}$.

Dentre os desafios a serem enfrentados pela ESF estão a formação de competências e habilidades com base nas relações partilhadas, como tecnologias leves de acolhimento, vínculo e responsabilização(8). Tais estratégias possibilitam ampliar o acesso às ações da saúde mental oferecidas à população em busca da integralidade da assistência. 
Ademais, a prática de promoção a saúde mental deve ser ampliada para toda a população territorial e não somente as PSM. A carência de investimento na formação dos trabalhadores (por meio da educação permanente em saúde) apresenta-se como lacuna perceptível para o melhor empoderamento na conduta aos usuários dessa ESF, característica evidenciada nas explanações da equipe, a partir das ações de prevenção do sofrimento mental e de promoção a saúde mental - inerentes ao campo da $\mathrm{APS}^{(9)}$.

Dessa forma, a ESF atua com o intuito de promover atenção à saúde ao exercer seu papel pautado nos princípios e diretrizes do Sistema Único de Saúde (SUS), com participação social a todos os usuários de uma população adstrita ${ }^{(7)}$. Configura-se no objetivo ao estímulo à promoção da saúde na construção da qualidade da assistência e desenvolvimento humano, envolvendo a solidariedade, interdisciplinaridade, articulação e comunicação entre os atores sociais (ESF e comunidade), para que unidos possam contribuir com a melhoria da qualidade de vida da população e não somente das PSM ${ }^{(9-}$ 10).

Destarte, além do acolhimento, a equipe deve estar preparada para desenvolver ações de socialização e prevenção dos possíveis agravos dos usuários da sua área de abrangência, como por exemplo, gerar e estimular atividades ou orientações em grupo com a população (adoecida ou não), seus familiares/ cuidadores, intervenções domiciliares para diminuição da sobrecarga da família cuidadora, utilização dos espaços públicos contidos no território para promoção da cidadania dos usuários e a abordagem de redução de danos ${ }^{(11)}$. Além do estímulo ao desevolvimento de Projeto Terapêutico Singular, o qual baseia-se em uma proposta de cuidado que busca suprir as demandas de saúde das pessoas que são assistidas pela ESF, mas sempre considerando a individualidade de cada usuário e, por isso, conta com a equipe multidisciplinar, articulando saberes, práticas e conhecimento dos dispositivos comunitários, possibilitando a inserção da população em seu próprio contexto social ${ }^{(12)}$.

\section{Categoria Temática 2 - Dificuldades e limitações para a implementação de ações de saúde mental na Estratégia Saúde da Família}

Na presente categoria, as falas convergiram para a falta de qualificação/capacitação dos trabalhadores em lidar com o sofrimento mental, uma vez que emergiram os seguintes elementos: o medo em conviver/lidar com o público em sofrimento mental e a confissão da incapacidade de implementar ações voltadas à promoção da saúde mental da população, que resultaram na dificuldade em gerar novas ações para os usuários da ESF acerca da promoção à saúde mental e prevenção de agravos.

Embora a maioria dos trabalhadores já tenha participado de algum curso de qualificação/capacitação profissional em saúde mental, eles relataram não possuir preparo suficiente para lidar com a PSM. Portanto, observar-se que o processo de educação permanente deve ser utilizado de forma mais concreta, como um dispositivo constante para os trabalhadores das equipes de saúde da família, a partir do contexto em que cada equipe encontra-se inserida.

Sob esse aspecto, é preciso que a gestão viabilize qualificação profissional em todos os níveis de atenção, de maneira a interagir com a PSM, em busca de ações articuladas de promoção e prevenção a partir de políticas públicas, por possibilitar a criação de uma prática intersetorial|(13).

As fragilidades inerentes aos processos de qualificação dificultam o trabalho das equipes em saúde mental, e comprometem o acompanhamento de mudanças propostas pela RPB nas três esferas de governo ${ }^{(14-15)}$.

A partir das fragilidades identificadas nos resultados desta pesquisa, faz-se necessário que a gestão oferte mais qualificações, associadas à mudança simultânea de postura da equipe em prol de ações pró-ativas nos processos formativos.

A fim de potencializar a construção do conhecimento que se encontra incipiente, torna-se necessário possibilitar aos trabalhadores o desenvolvimento de diversas habilidades, além das consideradas eminentes ao profissional de saúde, tais como: recepcionar, atender, escutar, dialogar, tomar decisão, amparar, orientar e negociar. Já que o cuidado caracteriza-se pelo processo no qual o trabalhador e a instituição tomam, para si, a responsabilidade de implementar ações intervencionistas em uma dada realidade na sua área de atuação, a partir das principais necessidades de saúde, em busca de uma 
relação acolhedora e humanizada para prover saúde nos níveis individuais e coletivo ${ }^{(16-17)}$.

Além disso, a construção do medo à PSM pode ser uma barreira que limita a equipe saúde da família em desenvolver atividades voltadas a tais usuários, ou até mesmo em agregá-los em ações conjuntas com a comunidade. Nessa perspectiva, estudos apontam que ainda na graduação, a PSM é representada a partir de uma imagem negativa de perigosa, com características amedrontadoras e deterioradas, trazidas da sua construção histórico-familiar e levadas para a academia e, se não desconstruída, tal imagem é levada para a vida profissional ${ }^{(16-17)}$.

Ao pensar numa resolutividade para a minimização das queixas referentes à falta de qualificação, a equipe saúde da família desenvolve rodas de conversas entre os usuários, familiares e trabalhadores da ESF, com o objetivo de buscar subsídios para a implementação de ações de saúde mental e encaminharam o planejamento à gestão. Tal situação pode ser visualizada como alternativa que podem sanar as lacunas que impedem a atenção integral à PSM ${ }^{(13)}$.

Salienta-se a necessidade de envolver as lideranças locais e representantes de familiares de PSM, como forma de controle social. Da mesma forma, é necessário o envolvimento dos órgãos formadores, para que a longo e médio prazo os profissionais saiam da sua respectiva formação com maior qualificação, para assumirem em seus processos de trabalho a produção do cuidado em saúde mental nos distintos equipamentos de atenção à saúde.

\section{Categoria Temática 3 - Estratégias/intervenções para implementação de saúde mental na Atenção Primária à Saúde}

Na presente categoria, a mais emblemática estratégia/intervenção que emergiu dos recortes das falas dos participantes da pesquisa foi o desenvolvimento da Roda de Terapia Comunitária (RTC), destacada como instrumento utilizado pela equipe saúde da família para a produção do cuidado em saúde mental. Nem mesmo as consultas individuais médicas e de enfermagem foram citadas e/ou identificadas com frequência, embora seja um espaço de diálogo e cuidado entre o profissional e o usuário, não só ao cuidado da saúde mental, mas para a saúde integral do indivíduo.

A terapia comunitária surgiu como um dispositivo de cuidado nos programas de inserção e apoio à saúde mental da população. É um espaço de acolhimento para a partilha de sofrimentos e sabedoria de vida, que ocorre de maneira circular e horizontal. Constitui um espaço de escuta, reflexão e compartilhamento de experiências, criando uma teia de relação social entre os participantes, na busca de soluções para os conflitos pessoais e familiares ${ }^{(18)}$.

A RTC é definida como a prática de cuidado de saúde em grupo. Consiste em reuniões para a escuta, acolhimento e partilha de problemas, no qual são expostas situações adversas na vida pessoal e/ou profissional que por diversas vezes são causadores de ansiedades e perdas de sono, podendo provocar agravos à sua saúde física e mental. Na RTC um indivíduo pode apoiar o outro no intuito da busca de soluções, nas trocas de experiências e pela identificação de vivências entre os participantes ${ }^{(19)}$.

A partir da RTC, é possível uma aproximação da equipe com a PSM, familiar e membros da comunidade, possibilitando a ampliação do vínculo e da terapêutica direcionada aos usuários.

Deste modo, pode-se afirmar que a RTC é um espaço de acolhimento do sofrimento, no qual os indivíduos se encontram e sentam-se lado a lado, para compartilhar seus anseios, dificuldades e situações do cotidiano, além de partilhar alegrias, vitórias e histórias de superação, ou seja, na RTC ocorre a escuta das histórias de vida das pessoas, gerando a relevância do saber de cada membro, adquirido pela própria experiência de vida ${ }^{(18-19)}$.

Face ao que foi discutido na categoria, observa-se que os profissionais evidenciaram a importância de se trabalhar com foco na saúde mental com todas as possibilidades terapêuticas que o serviço oferece, sejam elas dentro ou fora da unidade, com o intuito de abranger a promoção da saúde mental na APS, como preconizado pela RPB. No entanto, essa relevância citada nos discursos enfrenta as dificuldades da grande demanda de atendimento e, consequentemente, falta de tempo, bem como a insegurança da maioria em criar, implementar, gerenciar e lidar com a oferta de ações voltadas para a promoção da saúde mental e prevenção de agravos na atenção primária à saúde. 
Os resultados encontrados permitiram conhecer as percepções da equipe saúde da família acerca da implementação de ações de saúde mental na APS, através da limitação conceitual sobre o objeto deste estudo, uma vez que a maioria dos participantes evidenciou em suas falas a dificuldade em conceituar e descrever ações de promoção à saúde mental para os usuários da ESF.

Além disso, os profissionais relataram não possuir capacitações suficientes para lidarem com ações de saúde mental na APS e explanaram, de forma sucinta e pontual, sobre estratégias/intervenções, citando o acolhimento e a RTC como possibilidade de produção do cuidado em saúde mental no cenário dos serviços da ESF, embora suas percepções estivessem constantemente voltadas para o público já em sofrimento mental, reduzindo a possibilidade de promoção da saúde mental para todos os usuários do território da ESF em questão.

Tal visão reducionista contribui substancialmente para um olhar direcionado apenas à PSM, o que remete à necessidade de (re)pensar a inclusão de ações de prevenção do sofrimento mental e promoção da saúde em todo o território, reconhecendo-o como espaço de produção de subjetividades.

Diante dos resultados, acreditamos ser necessária a potencialização nos processos formativos dos trabalhadores dos serviços da ESF no campo da saúde mental, pautada nas diretrizes da Política Nacional de Educação Permanente em Saúde.

Portanto, a presente pesquisa alcançou seus objetivos propostos, na medida em que permitiu identificar o olhar de trabalhadores da ESF acerca da produção do cuidado em saúde mental e, concomitantemente, buscar a partir de tal ótica, as fragilidades e potencialidades/estratégias que permeiam as ações que constituem seus processos de trabalho. Ao mesmo tempo, a realização da pesquisa pode ser tomada como instrumento avaliativo, tanto para os trabalhadores, quanto para a gestão dos serviços de saúde, possibilitando aos distintos atores (re)pensarem a relevância da inserção da produção de subjetividades no processo de produção de cuidado à saúde como recurso essencial à integralidade da atenção.

\section{REFERÊNCIAS}

1. Maciel SC. Reforma psiquiátrica no Brasil: algumas reflexões. Cad. Bras. Saúde Mental. 2012; 4(8): 73 -82.

2. Reis HFT. Famílias de pessoas em sofrimento mental: um olhar fenomenológico sobre as relações de convivência [dissertação]. Jequié (BA): Universidade Estadual do Sudoeste da Bahia; 2010.

3. Jasnievski CR, Paes MR, Guimarães AN, Brusamarello T, Maftum MA. Tratamento da pessoa com transtorno mental em face da Reforma Psiquiátrica: percepções dos familiares. Colomb. Med. [Internet] 2011; 42(Suppl 2) [acesso em 15 set 2015]. Disponível: https://tspace.library.utoronto.ca/bitstream/1807/61320/1/rc11041.pdf.

4. Oliveira MD, Sá MF, Rocha ML. Percepção da sobrecarga familiar nos cuidados ao paciente psiquiátrico crônico. Enferm. foco. [Internet] 2011; 2(4) [acesso em 27 set 2015]. Disponível: http://revista.portalcofen.gov.br/ index.php/enfermagem/article/view/193/129.

5. dos Santos EM. Representações sociais sobre o cuidado em saúde mental na atenção básica: percepções de enfermeiros da estratégia saúde da família [tese]. Campinas (SP): Universidade Estadual de Campinas; 2014.

6. Minayo MCS. Pesquisa Social: teoria, método e criatividade. 29aa ed. Petrópolis: Vozes; 2010.

7. Ministério da Saúde (BR). Secretaria de Atenção à Saúde. Departamento de Atenção Básica. Cadernos da atenção básica: saúde mental. Brasília: Ministério da Saúde; 2013.

8. Amarante AL, Lepre AS, Gomes JLD, Pereira AV, Dutra VFD. As estratégias dos enfermeiros para o cuidado em saúde mental no programa saúde da família. Texto Contexto Enferm. 2011; 20(1): 85-93.

9. Merces AMF, Souza BML, da Silva TL, Silva TTM, Cavalcanti AMTS. Práticas de enfermagem em saúde mental na Estratégia de Saúde da Família: revisão integrativa. Cogitare Enferm. [Internet] 2015; 20(2) [acesso em 17 out 
2015]. Disponível: http://dx.doi.org/10.5380/ce.v20i2.38560.

10. Ministério da Saúde (BR). Portaria n 3.088, de 23 de dezembro de 2011: institui a Rede de Atenção Psicossocial para pessoas com sofrimento ou transtorno mental e com necessidades decorrentes do uso de crack, álcool e outras drogas, no âmbito do Sistema Único de Saúde. Diário Oficial da União, [Internet] 26 dez 2011 [acesso em 05 out 2015]. Disponível: http://bvsms.saude.gov.br/bvs/saudelegis/gm/2011/prt3088_23_12_2011_rep.html.

11. Tisott ZL, Hildebrandt LM, Leite MT, Martins RV, Cosentino SF. Álcool e outras drogas e a implantação da política de redução de danos no Brasil: revisão narrativa. Rev. de Atenção a Saúde. [Internet] 2015; 13(43) [acesso em 18 jan 2016]. Disponível: http://dx.doi.org/10.13037/rbcs.vol13n43.2730.

12. Pinto DM, Jorge MSB, Pinto AGA, Vasconcelos MGF, Cavalcante CM, Flores AZT, et al. Projeto terapêutico singular na produção do cuidado integral: uma construção coletiva. Texto Contexto Enferm. [Internet] 2011; 20(3) [acesso em 18 jan 2016]. Disponível: http://dx.doi.org/10.1590/S0104-07072011000300010.

13. Ribeiro LM, de Medeiros SM, de Albuquerque JS, Fernandes SMBA. Saúde mental e enfermagem na estratégia saúde da família: como estão atuando os enfermeiros?. Rev. esc. enferm. USP. [Internet] 2010; 2(44) [acesso em 15 ago 2015]. Disponível: http://dx.doi.org/10.1590/S0080-62342010000200019.

14. Arce VAR, de Sousa MF, Lima MG. A práxis da saúde mental no âmbito da estratégia saúde da família: contribuições para a construção de um cuidado integrado. Physis. 2011; 21(2): 541-60.

15. Ferreira-Furegato AR, Frari-Galera SA, Pillon SC, Ferreira-Santos JL, Araujo-Pitia AC, Cardoso L. Characterizing mental health care service teams. Rev. salud pública. 2010; 12(5): 732-43.

16. Ronchi JP, Avellar LZ. Saúde mental da criança e do adolescente: a experiência do Capsi da cidade de Vitória - ES. Psicol. teor. prat. 2010; 12(1): 71-84.

17. Villela JC, Maftum MA, Paes MR. O ensino de saúde mental na graduação de enfermagem: um estudo de caso. Texto Contexto Enferm. 2013; 22(2): 397-406.

18. Ferreira Filha MO, Lazarte R, Barreto AP. Impacto e tendências do uso da Terapia Comunitária Integrativa na produção de cuidados em saúde mental. Rev. Eletr. Enf. [Internet] 2015; 17(2) [acesso em 10 out 2015]. Disponível: http://dx.doi.org/10.5216/ree.v17i2.37270.

19. Buzeli CP, da Costa ALRC, Ribeiro RLR. Promoção da Saúde de Estudantes Universitários: Contribuições da Terapia Comunitária. G\&S. [Internet] 2012; 3(1) [acesso em 01 fev 2016]. Disponível: http://gestaoesaude.unb.br/ index.php/gestaoesaude/article/viewFile/151/pdf. 